\title{
Hipótese glutamatérgica da esquizofrenia: uma revisão integrativa de literatura
}

\author{
The glutamatergic hypothesis of schizophrenia: an integrative literature review \\ La hipótesis glutamatérgica de la esquizofrenia: una revisión integradora de la literatura
}

Recebido: 07/09/2021 | Revisado: 15/09/2021 | Aceito: 16/09/2021 | Publicado: 18/09/2021

Bárbara Queiroz de Figueiredo

ORCID: https://orcid.org/0000-0003-1630-4597

Centro Universitário de Patos de Minas, Brasil

E-mail: barbarafigueiredo@unipam.edu.br

Ana Luiza Costa Dias

ORCID: https://orcid.org/0000-0002-3496-4364

Universidade Itaúna, Brasil

E-mail: analuizacd2611@ outlook.com

Bruna Damas de Carvalho

ORCID: https://orcid.org/0000-0001-7770-3028

Centro Universitário de Goiatuba, Brasil

E-mail: brunadamascvlh@gmail.com

Gustavo Alves Medeiros

ORCID: https://orcid.org/0000-0002-6732-0258

Centro Universitário de Atenas, Brasil

E-mail: gustapo13m@gmail.com

Marcielle Francisca Capanema Santana

ORCID: https://orcid.org/0000-0002-5115-3352

Centro Universitário de Goiatuba, Brasil

E-mail: marciellecapanema@gmail.com

Matheus Lemes Baliano

ORCID: https://orcid.org/0000-0002-5420-037X

Universidade de Uberaba, Brasil

E-mail: matheusbaliano@gmail.com

Sarah Laís Penido Machado

ORCID: https://orcid.org/0000-0003-1153-8458

Universidade de Itaúna, Brasil

E-mail: sarahlaispenido@gmail.com

\begin{abstract}
Resumo
Introdução: A esquizofrenia é uma doença grave, episódica e persistente, com um curso de tempo característico em que episódios agudos, caracterizados por sintomas psicóticos positivos, como delírios e alucinações, são seguidos por uma fase crônica em que sintomas negativos e cognitivos incapacitantes e deficiências sociais tendem a ser proeminentes. O aminoácido glutamato é o principal neurotransmissor excitatório do sistema nervoso central (SNC), presente em cerca de 30 a $40 \%$ das sinapses cerebrais e em $80 \%$ das áreas envolvidas em processos cognitivos, principalmente no córtex cerebral e no hipocampo. Objetivo: evidenciar a hipótese glutamatérgica na fisiopatologia da esquizofrenia. Metodologia: Trata-se de uma revisão integrativa da literatura, e a pesquisa foi realizada através do acesso online nas bases de dados National Library of Medicine (PubMed MEDLINE), Scientific Electronic Library Online (Scielo), Google Scholar, Biblioteca Virtual em Saúde (BVS) e EBSCO Information Services, no mês de setembro de 2021. Resultados e discussão: A relação da neurotransmissão glutamatérgica com os sintomas apresentados por indivíduos esquizofrênicos pode ser validada ao avaliar a estreita interação entre os receptores NMDA de glutamato na via mesocortical, responsável pelas funções cognitivas normais e pela motivação, e a consequente liberação de dopamina. Em situações de hipofunção da via do glutamato, há pouca liberação de dopamina no córtex, o que resulta nos sintomas negativos e cognitivos. Conclusão: uma série de evidências sugere o envolvimento dos receptores glutamatérgicos tipo NMDA na esquizofrenia.
\end{abstract}

Palavras-chave: Esquizofrenia; Glutamato; NMDA; Fisiopatologia.

\begin{abstract}
Introduction: Schizophrenia is a severe, episodic and persistent disease with a characteristic time course in which acute episodes, characterized by positive psychotic symptoms such as delusions and hallucinations, are followed by a chronic phase in which disabling negative and cognitive symptoms and impairments social groups tend to be prominent. The amino acid glutamate is the main excitatory neurotransmitter of the central nervous system (CNS), present in approximately 30 to $40 \%$ of brain synapses and in $80 \%$ of areas involved in cognitive processes, mainly in the cerebral cortex and hippocampus. Objective: to demonstrate the glutamatergic hypothesis in the pathophysiology of schizophrenia. Methodology: This is an integrative literature review, and the search was
\end{abstract}


performed through online access in the National Library of Medicine (PubMed MEDLINE), Scientific Electronic Library Online (Scielo), Google Scholar, Virtual Health Library (BVS) and EBSCO Information Services, September 2021. Results and discussion: The relationship of glutamatergic neurotransmission with the symptoms presented by schizophrenic individuals can be validated by evaluating the close interaction between NMDA glutamate receptors in the mesocortical pathway, responsible for normal cognitive functions and motivation, and the consequent release of dopamine. In situations of hypofunction of the glutamate pathway, there is little release of dopamine in the cortex, which results in negative and cognitive symptoms. Conclusion: a lot of evidence suggests the involvement of NMDA-type glutamatergic receptors in schizophrenia.

Keywords: Schizophrenia; Glutamate; NMDA; Pathophysiology.

\section{Resumen}

Introducción: La esquizofrenia es una enfermedad severa, episódica y persistente con un curso temporal característico en el que los episodios agudos, caracterizados por síntomas psicóticos positivos como delirios y alucinaciones, son seguidos de una fase crónica en la que tienden los síntomas y deterioros cognitivos y negativos discapacitantes y los grupos sociales. ser prominente. El aminoácido glutamato es el principal neurotransmisor excitador del sistema nervioso central (SNC), presente en aproximadamente del 30 al $40 \%$ de las sinapsis cerebrales y en el $80 \%$ de las áreas involucradas en los procesos cognitivos, principalmente en la corteza cerebral y el hipocampo. Objetivo: demostrar la hipótesis glutamatérgica en la fisiopatología de la esquizofrenia. Metodología: Se trata de una revisión integradora de la literatura, y la búsqueda se realizó a través del acceso en línea en la Biblioteca Nacional de Medicina (PubMed MEDLINE), Scientific Electronic Library Online (Scielo), Google Scholar, Virtual Health Library (BVS) y EBSCO Information Services. Septiembre de 2021. Resultados y discusión: La relación de la neurotransmisión glutamatérgica con los síntomas que presentan los individuos esquizofrénicos se puede validar evaluando la estrecha interacción entre los receptores de glutamato NMDA en la vía mesocortical, responsables de las funciones cognitivas normales y la motivación, y la consiguiente liberación de dopamina. . En situaciones de hipofunción de la vía del glutamato, hay poca liberación de dopamina en la corteza, lo que resulta en síntomas negativos y cognitivos. Conclusión: mucha evidencia sugiere la participación de receptores glutamatérgicos de tipo NMDA en la esquizofrenia.

Palabras clave: Esquizofrenia; Glutamato; NMDA; Fisiopatología.

\section{Introdução}

As doenças mentais são consideradas doenças crônicas, que causam fragilidade e incapacidade para o portador, e são cercadas de estigmas e preconceitos de toda a sociedade. Nesse contexto das doenças mentais, a esquizofrenia é considerada a doença mais incapacitante quando nos referimos a transtornos mentais, e é definida pela Classificação Internacional de Doenças (CID-10) da Organização Mundial da Saúde (OMS) como distorções fundamentais e características do pensamento e da percepção, e por afetos inapropriados ou embotados, e envolvem fenômenos psicopatológicos como transtornos de pensamentos, ideias delirantes e vozes alucinatórias, por esse motivo o cuidado tornou-se ainda mais árduo, principalmente para o familiar responsável pelo cuidado (Dias, 2020).

A esquizofrenia é uma doença grave, episódica e persistente, com um curso de tempo característico em que episódios agudos, caracterizados por sintomas psicóticos positivos, como delírios e alucinações, são seguidos por uma fase crônica em que sintomas negativos e cognitivos incapacitantes e deficiências sociais tendem a ser proeminentes (Kumar et al. 2018). O entendimento atual da esquizofrenia é constituído pela hipótese dopaminérgica que denota alterações da neurotransmissão da dopamina no sistema mesolímbico responsável por sintomas positivos e via mesocortical, causando sintomas negativos, complementada pela hipótese glutamatérgica que considera mudanças na neurotransmissão neuronal pré-frontal envolvendo a conectividade glutamatérgica em NMDA receptor (Laruelle et al. 2014). Em particular, o aumento da síntese de dopamina présináptica é relevante para a patogênese da esquizofrenia (Howes et al. 2015). Os métodos de tratamento da esquizofrenia são classificados como o primeiro (principalmente antagonistas do receptor D 2 da dopamina), segundo (antagonistas multi-alvo com maior antagonismo na serotonina 5-HT 2Ado receptor de dopamina em D 2 do receptor) e antipsicóticos terceira geração representada, por exemplo, por aripiprazole, brexpiprazole e cariprazine. O aripiprazol é um agonista parcial do receptor D 2 da dopamina na via $\mathrm{G} \alpha$, mas pode exibir atividade agonista, agonista parcial ou antagonista no receptor D 2 da dopamina após 
diferentes leituras de sinalização. Em particular, é um antagonista ou agonista parcial da via de sinalização de $\beta$-arrestina-2 (Allen et al. 2011).

A hipótese dopaminérgica da esquizofrenia foi proposta pela primeira vez na década de 1960, quando a clorpromazina foi introduzida como o primeiro antipsicótico e provou tratar os sintomas positivos da doença. Posteriormente, a descoberta de que a anfetamina produz psicose foi outra prova do papel do excesso de dopamina na esquizofrenia. Propôs-se, então, que o aumento da neurotransmissão da dopamina poderia ser a causa dessa doença. $\mathrm{O}$ avanço de novos antipsicóticos estava de acordo com a hipótese dopaminérgica da esquizofrenia, pois foi observado que os sintomas positivos da doença podem ser atenuados com antagonistas do receptor de dopamina. No entanto, alguns achados contradizem essa hipótese, por exemplo, a clozapina, que é um antipsicótico muito eficaz em pacientes com esquizofrenia resistente, tem afinidade bastante baixa para a dopamina D 2receptores. Além disso, alguns pacientes com esquizofrenia também apresentam níveis normais de metabólitos de dopamina no líquido cefalorraquidiano ou no soro (Stepnicki et al. 2018).

O aminoácido glutamato é o principal neurotransmissor excitatório do sistema nervoso central (SNC), presente em cerca de 30 a 40\% das sinapses cerebrais e em $80 \%$ das áreas envolvidas em processos cognitivos, principalmente no córtex cerebral e no hipocampo. Dentre inúmeros mecanismos fisiológicos, o glutamato tem participação no desenvolvimento neural, plasticidade sináptica, funções de aprendizado e memória. Ademais, exerce sua ação pela interação com receptores específicos nos neurônios pós-sinápticos, e o controle da neurotransmissão é feito por transportadores específicos, uma vez que o glutamato não possui uma enzima que o degrade, como ocorre com outros neurotransmissores (Yota et al. 2019). Sob essa perspectiva, o objetivo deste estudo é evidenciar a hipótese glutamatérgica na fisiopatologia da esquizofrenia.

\section{Metodologia}

Trata-se de uma pesquisa descritiva do tipo revisão integrativa da literatura, que buscou evidenciar a hipótese glutamatérgica na fisiopatologia da esquizofrenia. A pesquisa foi realizada através do acesso online nas bases de dados National Library of Medicine (PubMed MEDLINE), Scientific Electronic Library Online (Scielo), Cochrane Database of Systematic Reviews (CDSR), Google Scholar, Biblioteca Virtual em Saúde (BVS) e EBSCO Information Services, no mês de setembro de 2021. Para a busca das obras foram utilizadas as palavras-chaves presentes nos descritores em Ciências da Saúde (DeCS): em inglês: "schizophrenia", "glutamate", "NMDAR", "dopamine", "physiopathology", em espanhol: "esquizofrenia", "glutamato", "NMDAR", "dopamina", "fisiopatologia" e em português: "esquizofrenia", "glutamato", "NMDAR", "dopamina", "fisiopatologia".

Como critérios de inclusão, foram considerados artigos originais, que abordassem o tema pesquisado e permitissem acesso integral ao conteúdo do estudo, publicados no período de 2000 a 2021, em inglês, espanhol e português. O critério de exclusão foi imposto naqueles trabalhos que não estavam em inglês, português e espanhol que não tinham passado por processo de Peer-View e que não relacionassem o glutamato à fisiopatologia da esquizofrenia. A estratégia de seleção dos artigos seguiu as seguintes etapas: busca nas bases de dados selecionadas; leitura dos títulos de todos os artigos encontrados e exclusão daqueles que não abordavam o assunto; leitura crítica dos resumos dos artigos e leitura na íntegra dos artigos selecionados nas etapas anteriores. Assim, totalizaram-se 29 artigos científicos para a revisão integrativa da literatura, com os descritores apresentados acima.

\section{Resultados e Discussão}

Estudos post-mortem de cérebros de pacientes esquizofrênicos demonstraram alterações na densidade de receptores glutamatérgicos no córtex pré-frontal, tálamo e lobo temporal, demonstrando que essas áreas cerebrais apresentaram ativação 
diminuída durantes testes de performance nos pacientes (Meador-Woodruff, 2000). Além disso, demonstrou-se que pacientes com esquizofrenia apresentavam diminuição na concentração de glutamato no líquido cefalorraquidiano, achados esses que compreendem evidencias da hipofunção glutamatérgica na esquizofrenia (Celotto et al. 2019).

A hipótese da dopamina pode ser responsável por certos aspectos da psicopatologia da esquizofrenia, especialmente os sintomas positivos (Yui et al. 200)). No entanto, com a possível exceção da clozapina, os antipsicóticos têm efeitos desprezíveis sobre os sintomas negativos e cognitivos, os mais robustos preditores de incapacidade na esquizofrenia (Lim et al. 2016). Além disso, a atrofia cortical se correlaciona com sintomas negativos e cognitivos na esquizofrenia crônica, mas não com a gravidade da psicose. Assim, as características centrais da esquizofrenia, que são as principais responsáveis pela incapacidade persistente, estão ligadas à patologia cortical generalizada e são improváveis como consequência da simples disfunção da dopamina (Coyle et al. 2018).

Os medicamentos atuais para o tratamento da esquizofrenia - os antipsicóticos típicos e atípicos - são antagonistas do receptor D2 da dopamina com um benefício clínico satisfatório nos sintomas positivos, mas sem impacto limitado ou nenhum nos sintomas negativos da doença (Allen et al. 2011). Portanto, há uma necessidade urgente de melhorar a farmacoterapia dos sintomas negativos. Ao lado do sistema dopaminérgico, a neurotransmissão glutamatérgica disfuncional tem sido fortemente implicada na etiologia da esquizofrenia, particularmente uma hipofunção do $\mathrm{N}$ - receptor de metil-d-aspartato (NMDAR) (Borba et al. 2017), conforme ilustrado pela Figura 1. Como a hipofunção NMDAR está especialmente associada a sintomas negativos, o NMDAR é continuamente discutido como um alvo promissor para a introdução de novos medicamentos (Yota et al. 2019).

Figura 1: Estrutura do receptor $\mathrm{N}$-metil-d-aspartato (NMDAR; GluN1 / GluN2). O NMDAR convencional compreende um complexo heterotetramérico de duas subunidades GluN1 e duas GluN2. ATD, domínio do terminal amino; CTD, domínio do terminal carboxi; GMS, sítio modulador de glicina; LBD, domínio de ligação ao ligando; PCP, fenciclidina; TMD, domínio transmembranar.

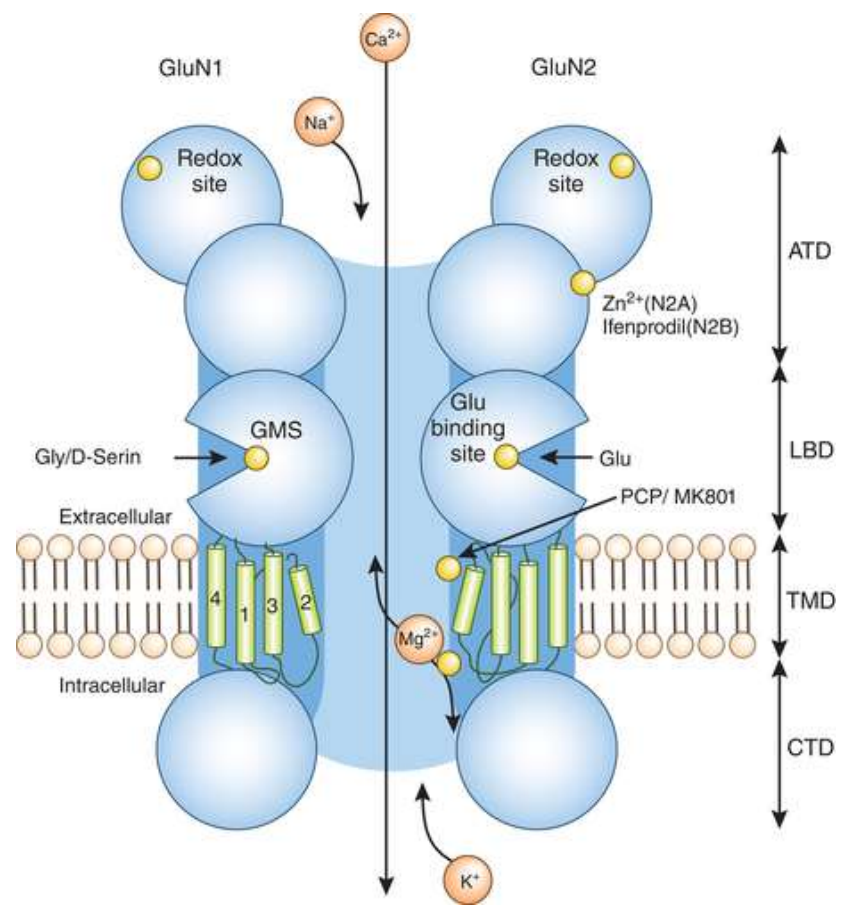

Fonte: Yota et al. (2019). 
Doses subanestésicas de antagonistas NMDAR, como fenciclidina (PCP) e cetamina, não provocam apenas efeitos psicotomiméticos que lembram sintomas positivos, negativos e cognitivos da esquizofrenia em voluntários saudáveis, mas também exacerba os sintomas em pacientes com esquizofrenia (Björkholm et al. 2017). Portanto, a sinalização NMDAR reduzida pode ser uma rota única para modelar sintomas negativos reminiscentes de esquizofrenia (Beck et al. 2016). Assim, investigar os efeitos da cetamina em voluntários saudáveis pode ser especialmente útil para descrever os mecanismos neurofisiológicos associados aos sintomas negativos. Para ativar o NMDAR, não só a ligação do glutamato, mas também da glicina é essencial. Os ensaios clínicos de agonistas do local da glicina (por exemplo, a própria glicina, d-serina) ou o inibidor da recaptação da glicina sarcosina em pacientes com esquizofrenia crônica relataram efeitos benéficos nos sintomas negativos da esquizofrenia (Thiebes et al. 2017).

Mismatch negativity (MMN) é um potencial auditivo relacionado a eventos (ERP) gerado quando um estímulo desviante é apresentado que difere em algumas características físicas, como duração ou frequência, de estímulos padrão repetidamente apresentados (González-Maeso et al. 2008). Amplitudes reduzidas de MMN têm se mostrado um achado robusto em pacientes com esquizofrenia em vários estudos (Howes et al. 2015). Sugere-se que os déficits de negatividade incompatíveis, também conhecidos como sinais de erro de previsão, são particularmente graves em pacientes com esquizofrenia crônica que apresentam sintomas negativos proeminentes. Além disso, presume-se que a atividade neural subjacente ao MMN pode ser atribuída a dois conjuntos de geradores neurais (Herman et al. 2012).

Os geradores temporais superiores estão associados à parte da memória sensorial da detecção de mudanças, e os geradores frontais são supostamente responsáveis por desencadear uma mudança de atenção na detecção de mudanças (Menniti et al. 2013). Além das amplitudes do MMN, a densidade da fonte de corrente (CSD) dos geradores temporais e frontais do MMN mostrou ser reduzida em pacientes com esquizofrenia em comparação com controles saudáveis. Além disso, uma redução da amplitude do MMN sugere uma associação com a neurotransmissão NMDAR glutamatérgica. Vários estudos eletrofisiológicos relataram reduções induzidas pela cetamina nas amplitudes do MMN (Thiebes et al. 2017).

Pacientes com história documentada de sintomas positivos proeminentes de esquizofrenia, mas atualmente sem apresentar sintomas positivos substanciais, foram considerados como satisfazendo os critérios da CID-10 para esquizofrenia residual se exibissem sintomas negativos apreciáveis e funções ocupacional ou social prejudicadas. A maioria dos pacientes estava recebendo medicação psicotrópica. A mediana de dose diária necessária foi calculada separadamente para antipsicóticos, estabilizadores de humor, incluindo lítio e antidepressivos (Kumar et al. 2018).

Não foram encontradas evidências sólidas do prolongamento da latência do MMN na condição de cetamina. No entanto, uma latência atrasada do MMN é relatada de forma menos consistente do que a redução da amplitude. Nos estudos, a Escala de Síndrome Positiva e Negativa (PANSS) foi usado para a investigação de sintomas semelhantes à esquizofrenia induzida por cetamina em pessoas saudáveis, embora tenha sido inicialmente projetado e validado para a avaliação de sintomas em pacientes com esquizofrenia (Matosin et al. 2014). No entanto, há evidências de uma semelhança nas dimensões dos sintomas entre os sintomas induzidos pela cetamina e a psicose da esquizofrenia, com a sobreposição mais consistente para o fator de sintoma negativo. Consequentemente, o PANSS foi usado em vários estudos recentes que investigaram sintomas semelhantes à esquizofrenia induzida por cetamina em voluntários saudáveis (Thiebes et al. 2017).

À primeira vista, os resultados com controles saudáveis parecem entrar em conflito com a conclusão de que a MMN não se correlaciona com sintomas clínicos em pacientes com esquizofrenia. Além disso, havia muitos participantes diferentes incluídos, desde pacientes com primeiro episódio de esquizofrenia crônica (Gao et al. 2000). Os estudos que relataram um MMN frontal menor com maiores sintomas negativos incluíram apenas pacientes com doença crônica, enquanto estudos com pacientes com esquizofrenia no primeiro episódio relataram correlações na direção reversa (Goff et al. 2000). Supondo que o MMN seja dependente da transmissão NMDAR, isso sugere que o papel do funcionamento do NMDAR se altera entre os 
diferentes estágios (Kumar et al. 2018). Além disso, ao avaliar a média de entrevistados que não aderiram ao uso de medicamentos, aproximadamente $40 \%$, ao considerar todas as implicações da não adesão ao portador de transtorno mental, como piora do prognóstico, crises mais frequentes e intensas, aumento do risco de tentativa de suicídio e comprometimento da qualidade de vida, conclui-se que é uma estatística preocupante (Martínez et al. 2015).

Glutationa e glutamato foram significativamente correlacionados entre si em todas as três regiões do cérebro (Jackson et al. 2012). Essas altas correlações são consistentes com a hipótese de uma ligação mecanicista entre os sistemas antioxidante e glutamatérgico no cérebro humano, de modo que, em condições de estado estacionário, baixos níveis de glutationa estão associados a baixos níveis de neurotransmissão glutamatérgica (Madeira et al. 2018). A princípio, o uso do sinal de água não suprimido como referência para a normalização do sinal do metabólito pode introduzir correlações espúrias entre os sinais de glutationa e glutamato (Stone et al. 2007). No entanto, desde que as variações no teor de água dentro da amostra sejam muito menores do que as variações nos níveis de metabólitos, a variação compartilhada entre os sinais de metabólitos normalizados pode ser considerada como originada em grande parte da variação compartilhada nas concentrações de metabólitos (Lin et al. 2019).

Porém, ainda são poucos os estudos que exploram a associação entre amplitudes reduzidas de MMN devido à administração de cetamina e sintomas negativos induzidos por cetamina em voluntários saudáveis (Uno et al. 2019). Os resultados indicam que o surgimento de sintomas negativos em indivíduos saudáveis após a administração de cetamina está associado a alterações cerebrais, avaliadas pelo MMN (Kaiser et al. 2005). Consequentemente, o MMN poderia representar potencialmente um biomarcador para sintomas negativos de esquizofrenia eliciados por funcionamento insuficiente de NMDAR (Tomaka et al. 2017). Assim, investigar os efeitos da cetamina em indivíduos saudáveis pode ser particularmente útil para a identificação de biomarcadores que podem permitir a previsão da resposta ao tratamento a substâncias que aumentam a função de NMDAR em pacientes com esquizofrenia com sintomas negativos proeminentes (Thiebes et al. 2017).

\section{Conclusão}

Nota-se, portanto, que elucidar o circuito patológico na esquizofrenia fornece novos alvos para intervenções terapêuticas. Em primeiro lugar, dado que a desinibição da entrada dopaminérgica estriatal, que se correlaciona com a psicose, é uma consequência do aumento da produção glutamatérgica do córtex cerebral, não é surpreendente que os antipsicóticos bloqueadores de D2R tenham pouco impacto sobre os sintomas negativos e cognitivos determinados corticamente. Em segundo lugar, estudos pré-clínicos com um modelo genético de esquizofrenia indicam que restaurar a função NMDAR corrigindo déficits de D-serina ou aumentando a responsividade NMDAR com um mGlu 5PAM reverte a patologia do tipo esquizofrênica.

Uma série de evidências sugere o envolvimento dos receptores glutamatérgicos tipo NMDA na esquizofrenia, bem como que antagonistas do receptor NMDA causam um quadro clínico que muito se assemelha à esquizofrenia, incluindo sintomas positivos, negativos e desagregação do pensamento. Ensaios clínicos com agentes glicinérgicos (co-agonistas NMDA) associados a antipsicóticos têm demonstrado eficácia no tratamento de sintomas negativos da esquizofrenia. Estes achados são a base da hipótese de hipofunção de receptores NMDA na esquizofrenia. É necessário o esclarecimento desta hipótese in vivo em pacientes com esquizofrenia.

\section{Referências}

Allen, J. A., et al. (2011). Descoberta de ligantes D2 de dopamina polarizados por $\beta$-arrestina para sondar as vias de transdução de sinal essenciais para a eficácia antipsicótica. Proc. Natl. Acad. Sci., 108 (8), 18488-18493.

Beck, K., et al. (2016). Targeting glutamate to treat schizophrenia: lessons from recente clinical studies. Revist Psychopharmacology, 233 (1), $2425-2428$. 
Björkholm, C., Marcus, M. M., Konradsson-Geuken, Å., Jardemark, K., Svensson, T. H. (2017). O novo antipsicótico brexpiprazol, sozinho e em combinação com escitalopram, facilita a transmissão glutamatérgica pré-frontal por meio de um mecanismo dependente do receptor D1 da dopamina. Neuropsychopharmacol., 27 (8), 411-417.

Borba, L. O., et al. (2017). Adesão do portador de transtorno mental à terapêutica medicamentosa no tratamento em saúde mental. Revista da Escola de Enfermagem da Universidade de São Paulo, 52 (3), 1-10.

Celotto, A. C., Durigin, L., Calfi, G. S. \& Rosa, M. L. N. M. (2019). Participação dos receptores metabotrópicos de glutamato e da via de sinalização por óxido nítrico no desenvolvimento da esquizofrenia. Manuscripta Médica, 2 (3), 1-15.

Coyle, J. T. \& Balu, D. T. (2018). O papel da serina racemase na fisiopatologia das doenças cerebrais. Adv. Pharmacol., 82 (8), 35 - 56.

Dias, P., et al. (2020). Bem-estar, qualidade de vida e esperança em cuidadores familiares de pessoas com esquizofrenia. Revista Portuguesa de Enfermagem de Saúde Mental, 17 (23), 23-30.

Gao, X. M., Sakai, K., Roberts, R. C., Conley, R. R. \& Dean, B. (2000). CA ionotrópicos receptores de glutamato e expressão de $N$-metil- $D$ subunidades do receptor aspartato em sub-regiões do hipocampo humano: Efeitos da esquizofrenia. Sou. J. Psychiatry, 157 (5), 1141-1149.

Goff, D. C. (2000). Glutamate receptores em esquizofrenia e drogas antipsicóticas. Em receptores de neurotransmissores em ações de medicamentos antipsicóticos. Lidow, 4 (8), 126-141.

González-Maeso, J., et al. (2008). Identificação de um complexo receptor de serotonina / glutamato implicado na psicose. Nature, 452 (8), 93-97.

Herman, E. J., Bubser, M., Conn, P. J. \& Jones, C. K. (2012). Metabotropic glutamate receptors for new treatment in esquizofrenia. Handb. Exp. Pharmacol., $8(1), 297-365$

Howes, O., et al. (2015). Glutamate and dopamine in schizophrenia: na update for the 21 century. Journal Psychopharmacol, 29 (2), 97-115.

Jackson, J., et al. (2012). Uma dieta sem glúten em pessoas com esquizofrenia e anticorpos anti-transglutaminase tecidual ou anti-gliadina. Esquizofr. Res., 140 (7), 262-263.

Kaiser, L. G., Schuff, N., Cashdollar, N. \& Weiner, M. W. (2005). Age-related glutamate and glutamine concentração changes in normal human brain: 1H MR espectroscopy study at 4 T. Neurobiol. Envelhecimento, 26 (8), 665-672.

Kumar, J., et al. (2018). Glutationa e glutamato na esquizofrenia. Revista Psiquiatria Molecular, 25 (1), 873-882.

Laruelle, M. (2014). Schizophrenia: From dopaminergic to glutamatérgic intervenções. Curr. Opiniões Pharmacol., 14 (8), 97-102.

Lim, J. et al. (2016). A relação entre subdomínios de sintomas negativos e cognição. Psychol. Med., 46 (4), 2169 - 2177.

Lin, C. H. \& Lane, H. Y. (2019). Early Identification and Intervention of Schizophrenia: Insight from Hypotheses of Glutamate Disfunction and Oxidative Stress. Revist Frontiers in Psychiatry, 27 (10), 1-9.

Madeira, C., et al. (2018). Níveis de glutamato e glutamina no sangue no início recente e na esquizofrenia crônica. Revista Fronteiras em Psiquiatria, 9 (173), $1-8$.

Martínez, C. J. \& Roman, V. R. (2015). Propuesta de un modelo de respuesta de los delírios esquizofrénicos a los antipsicóticos. Revista Associação Espanhola de Neuropsiquiatria, 36 (129), 15-28.

Matosin, N., Fernandez-Enright, F., Frank, E., Deng, C., Wong, J., Huang, X.-F. \& Newell, K. A. (2014). Receptor de glutamato metabotrópico mGluR2 / 3 e mGluR5 ligando-se ao córtex cingulado anterior em depressão psicótica e não psicótica, transtorno bipolar e esquizofrenia: implicações para novas terapêuticas baseadas em mGluR. J. Psychiatry Neurosci., 39 (8), 407-416.

Meador-Woodruff, J. H. (2000). Healy, expressão do receptor DJ Glutamato no cérebro esquizofrênico. Brain Res. Brain Res. Rev., 31(8), $288-294$.

Menniti, F. S., Lindsley, C. W., Conn, P. J., Pandit, J. \& Zagouras, P. (2013). Volkmann, RA Allosteric modulators for the treatment of esquizofrenia: Targeting glutamatérgic networks. Curr. Principal. Med. Chem., 13 (4), $26-54$.

Stepnicki, P., Kondej, M. \& Kaczor, A. A. (2018). Conceitos e tratamentos atuais da esquizofrenia. Molecules, 23 (8), 1-9.

Stone, J. M., Morrison, P. D. \& Pilowsky, L. S. (2007). Glutamato e desregulação da dopamina na esquizofrenia - A síntese e revisão seletiva. J. Psychopharmacol. Oxf. Engl., 21 (8), 440-452.

Thiebes, S., et al. (2017). Glutamatergic deficit and negative schizophrenia type: new evidence of incompatible and ketamine-induced negative changes in healthy men. Journal Psychiatry Neuroscience, 42 (4), 273-283.

Tomaka, J., et al. (2017). Gluten-related transtornos e esquizofrenia-potencial linking engines, diagnostic andapeutic challenge. Curr. Probl. Psychiatry.,18 (7), 9-24.

Uno, Y., et al. (2019). Glutamate hypothesis in schizophrenia. Psychiatry and Clinical Neurosciences, 73 (1), 204-215.

Yui, K., Ikemoto, S., Ishiguro, T. \& Goto, K. (2000). Estudos de psicose por anfetamina ou metanfetamina no Japão: Relação da psicose por metanfetamina com a esquizofrenia. Ann. NY Acad. Sci., 914 (3),1 - 12. 